\title{
The Emergence and Interpretation of
}

\section{Probability in Bohmian Mechanics ${ }^{1}$}

The Bohm interpretation of quantum mechanics is capable of illustrating, by itself, virtually every philosophical and foundational conundrum associated with physical probability. One reason this is true is that the Bohm interpretation comes in many forms, both stochastic and deterministic. The other reason is that quantum mechanics is to Bohmian mechanics roughly as statistical mechanics is to classical mechanics; hence the notorious problems in the foundations of statistical mechanics are reprised within the foundations of Bohmian mechanics. The present paper is an opinionated survey of this literature. In it I focus on the meaning of Born's rule in a Bohmian universe. After considering various rationales for this rule, I settle on one based on the law of large numbers as the best bet. This option leaves open how the probabilities in this result ought to be interpreted, or so I shall argue. When delivering an interpretation of these probabilities, the history of probability warns us of a number of pitfalls. I show how these pitfalls manifest themselves in the Bohmian case and then show that at least one class of interpretations seems to successfully navigate these problems.

\section{Bohmian Mechanics and the Distribution Postulate}

Bohmian mechanics is an empirically adequate and logically consistent quantum theory of non-relativistic phenomena. To my mind it provides the most natural answer to the notorious measurement problem. The measurement problem tells us that the wave function evolving according to the Schrödinger equation either can't be correct or it can't be the whole story. Collapse theories answer the problem by denying the evolution is always via the Schrödinger equation. Bohmian theories answer by denying the completeness of the wavefunction description of the world - there are also particles, or "beables" in Bell's terminology. Originally devised by de Broglie in 1927, it was subsequently rediscovered and improved by Bohm in 1952. More recently, many researchers have developed the approach further, especially the group led by Dürr, Goldstein and Zanghi (DGZ). ${ }^{2}$ For the purposes of this article, we shall limit ourselves to a brief sketch of the non-relativistic theory. The reader is encouraged to turn to the above references for treatments of spin, identical particles, quantum field theory, and more.

Bohmian mechanics is a theory of $\mathrm{N}$ point particles evolving in $\mathbb{R}^{3}$. Each particle (indexed by i) obeys a first-order equation of motion:

\footnotetext{
${ }^{1}$ Many thanks for comments and discussions are due to Jeff Barrett, Detlef Dürr, Sheldon Goldstein, Roman Frigg, Carl Hoefer, Barry Loewer and Tim Maudlin.

${ }^{2}$ For a selection of their work, the reader can visit www.bohmian-mechanics.net for papers and references. Tumulka 2004 is a useful introduction to Bohm's theory, and DGZ 1992 is a rigorous development of the theory.
} 


$$
\frac{d x_{i}}{d t}=\frac{\hbar}{m_{i}} \operatorname{Im} \frac{\nabla_{i} \Psi\left(x_{1}(t) \ldots x_{N}(t)\right)}{\Psi\left(x_{1}(t) \ldots x_{N}(t)\right)}
$$

where 'Im' means imaginary part, $m$ is the mass of the particle, and $\Psi$ is a timedependent, complex-valued function on configuration space $\mathbb{R}^{3 \mathrm{~N}}$. The wave function $\Psi$ evolves according to the familiar Schrödinger equation:

$$
i h \frac{\partial \Psi}{\partial t}=-\sum_{i=1}^{N} \frac{\hbar^{2}}{2 m_{i}} \nabla_{i} \cdot \nabla_{i} \Psi+V\left(q_{1} \ldots q_{N}\right) \Psi
$$

where $\mathrm{V}$ is the potential energy. Here $q_{i}$ are the variables in $\mathbb{R}^{3 \mathrm{~N}}$ upon which $\Psi$ depends. The system given by (1) and (2) describes a deterministic theory of the universe, one for which global existence and uniqueness can be proved for almost all initial conditions. Relevant to us later, the theory is also time-reversal invariant and (for most states) Poincaré recurrent. That is, for every history there is also the time-reversed history given by the transformations $t \rightarrow-t$ and $\Psi \rightarrow \Psi^{*}$; and for every initial condition $\left(\mathrm{x}_{1}(0) . . \mathrm{x}_{\mathrm{N}}(0), \Psi(0)\right)$, the time development by (1) and (2) will eventually take the system arbitrarily close to this state again.

So far, nothing has been said of probabilities, yet the empirical heart of quantum mechanics is the fact that subsystems of the universe (for instance, actual ensembles measured in labs) are probabilistically distributed according to Born's rule. The probability density for finding particles in configuration $q \equiv\left(q_{1} \ldots q_{N}\right)$ at time t, i.e., $\rho(q, t)$, is equal to $|\Psi(\mathrm{q}, \mathrm{t})|^{2}$. To be as successful as ordinary quantum mechanics, Bohmian mechanics must provide a reason why betting in accord with Born's rule is a rational strategy. That is, the Bohmian must explain why particles upon measurement actually will be distributed according to $|\Psi(\mathrm{q}, \mathrm{t})|^{2}$. The Bohmian needs to explain the origin of Born's rule.

Care needs to be taken in understanding Born's rule in a Bohmian world. The wavefunction $\Psi$ in (2) is the wavefunction of the universe, whereas the wavefunction empirically tested by Born's rule is a function of a subsystem of the universe. This distinction is important for what follows. Fortunately, the Bohmian can speak of a subsystem or effective wavefunction $\psi$ when - roughly - enough "decoherence" has occurred between the system and its environment (see DGZ 1992). ${ }^{3}$ The Bohmian will

\footnotetext{
${ }^{3}$ Let $\mathrm{Q}_{\mathrm{t}}$ be the actual configuration of particles in the universe at a time. In a composite system $Q_{t}=\left(X_{t}, Y_{t}\right)$, where $X$ is the actual subsystem of interest and $Y$ is the actual environment. Then a natural definition for a subsystem's wavefunction is what DGZ call a conditional wavefunction $\psi_{\mathrm{t}}(\mathrm{x})=\Psi_{\mathrm{t}}\left(\mathrm{x}, \mathrm{Y}_{\mathrm{t}}\right)$, where we calculate the universal wavefunction in the actual configuration of the environment. The conditional wavefunction will not in general evolve according to the Schrödinger equation. However, when certain further conditions are met (corresponding to the universal wavefunction evolving into a wide separation of components in the configuration space
} 
thus understand Born's rule in terms of $\psi$, not $\Psi$. Since position measurements reveal the positions of actual particles for the Bohmian, the success of Born's rule implies that the Bohmian particle positions of measured subsystems of the universe be randomly distributed according to $|\psi(\mathrm{q}, \mathrm{t})|^{2}$. Thus, the Bohmian must assume, derive or otherwise make plausible the claim that the position probability density is given by

$$
\rho(\mathrm{q}, \mathrm{t})=|\psi(\mathrm{q}, \mathrm{t})|^{2}
$$

whenever a measurement is made. Let's call (3) the distribution postulate. With (3) the Bohmian can explain the occurrence of frequencies in accord with Born's rule and all that follows from that, e.g., why quantum mechanics works, why the uncertainty principle holds, why the 'no superluminal signaling' theorem obtains, and more. Without (3) or something close to (3), the Bohmian runs a serious explanatory deficit.

The project of justifying (3) is often packaged as being very simple due to the following mathematical fact. The Schrödinger equation implies a continuity equation for $|\Psi|^{2}$. If we substitute $\rho(\mathrm{q}, \mathrm{t})=|\Psi(\mathrm{q}, \mathrm{t})|^{2}$ into the continuity equation then we get

$$
\frac{\partial \rho}{\partial t}+\frac{\partial(\rho \dot{x})}{\partial x}=0
$$

Equation (4) will take any initial distribution of particles such that $\rho(q, 0)=|\Psi(q, 0)|^{2}$ holds and evolve it in time $t$ to the distribution $\rho(q, t)=|\Psi(q, t)|^{2}$. The dynamics, in other words, preserves the probability distribution $\rho=|\Psi|^{2}$. The project of justifying (3) is then advertised as simply the problem of finding some reason to think $\rho(q, t)=|\Psi(q, t)|^{2}$ at some time or other, for one is then guaranteed that it will always hold.

However, despite being repeated many times, this way of putting matters is misleading. ${ }^{4}$ What we want explained is why a system of particles should have a probability density given by the effective wavefunction, not the universal wavefunction. The effective wavefunction is clearly the relevant object in Bohmian mechanics, for it is the object corresponding to the wavefunction in the usual use of Born's rule within orthodox quantum measurement theory. Yet the effective wavefunction does not always obtain. It evolves according to the Schrödinger equation when it exists, but the conditions for its existence do not always obtain. So we cannot make use of the above guarantee. That the dynamics preserves $\rho=|\Psi|^{2}$ does not imply that the dynamics preserves $\rho(\mathrm{q}, \mathrm{t})=|\psi(\mathrm{q}, \mathrm{t})|^{2}$.

of the entire system--see DGZ for details) it will evolve according to the Schrödinger equation. When this is the case it is called an effective wavefunction. Note that an effective wavefunction obtains whenever the orthodox formalism assigns a wavefunction. ${ }^{4}$ Thanks go to Tim Maudlin and Sheldon Goldstein for this point. The primary reason for this mistake, I suspect, is that prior to DGZ 1992 Bohm's theory was not rigorously presented as a universal theory. In hindsight, Bohmians were unwittingly talking about universal wavefunctions when they should have been talking about effective wavefunctions. 
The preservation of $\rho=|\Psi|^{2}$ is relevant and perhaps is a necessary condition for (3) obtaining, but it is not sufficient.

\section{The Analogy with Classical Statistical Mechanics}

Einstein famously wanted the statistical aspects of quantum theory to take "an approximately analogous position to the statistical mechanics within the framework of classical mechanics" (1949, p. 672). Since Bohmian mechanics is a deterministic and more fundamental theory than quantum mechanics, it is in the perfect position to realize this goal. Let's see how, first by comparing Bohmian mechanics with classical mechanics, and then by comparing quantum mechanics (as seen through Bohmian eyes) with classical statistical mechanics.

Classical mechanics is also a theory of $\mathrm{N}$ point particles evolving on $\mathbb{R}^{3}$. In contrast to Bohmian mechanics, wherein one requires an initial wavefunction and initial locations, here one requires an initial position and initial momentum. Given these values, Hamilton's two equations, the counterparts of (1) and (2), will determine a trajectory for an individual system. Like Bohmian dynamics, Hamilton's equations are also deterministic, time-reversal invariant and recurrent.

For an ensemble of systems evolving according to the same Hamiltonian, Hamilton's equations define a velocity field on phase space. This field evolves a distribution $\rho_{c}(x, p, t)$ via a continuity equation

$$
\frac{\partial \rho_{c}}{\partial t}+\frac{\partial\left(\rho_{c} \dot{q}\right)}{\partial q}+\frac{\partial\left(\rho_{c} \dot{p}\right)}{\partial p}=0
$$

which may be compared with (4). And just as $\rho_{c}(x, p, t)$ moves through phase space as an incompressible fluid due to Liouville's theorem, so does the distribution $\mathrm{f}(\mathrm{q}, \mathrm{t})=\rho(\mathrm{q}, \mathrm{t}) /|\psi|^{2}$ in Bohmian mechanics.

Taking the analogy further, note that in the classical case the uniform distribution is preserved by the dynamics, just as (3) is by the Bohmian dynamics. That is, (5) will evolve $\rho_{c}(x, p, 0)=$ constant in time $t$ to $\rho_{c}(x, p, t)=$ constant. Since one usually thinks of $\rho_{c}(x, p, t)=$ constant on the energy surface in phase space as the signature of thermal equilibrium, it is said that the classical dynamics leaves thermal equilibrium invariant. For this reason, advocates of Bohmian mechanics often think of the $\rho=|\psi|^{2}$ distribution as "quantum equilibrium." Like thermal equilibrium, it is preserved by the dynamics. (Or more accurately, the distribution at the level of the universal wavefunction is preserved by the dynamics; the distribution at the level of the effective wavefunction will be preserved when the effective wavefunction exists.) Unlike thermal equilibrium, which is stationary, quantum equilibrium is time dependent. As $\psi$ evolves, so does $\rho$. Nevertheless, the functional form between the two remains invariant. Bohmian mechanics call this close cousin to stationarity equivariance. The other disanalogy is that 
for the Bohmian, the universe is in, and perhaps always has been in, the quantum heat death, whereas we are not yet in the thermodynamic heat death.

Finally, observe that quantum equilibrium and thermodynamic equilibrium are independent of one another. One can be in the first and not the second, and the second without the first. Thermodynamic equilibrium is first and foremost a feature of the wave function of the universe.

\section{Why Justify the Distribution Postulate?}

The status of the distribution postulate has been controversial since Bohm announced his theory. Pauli 1953 and Keller 1953 soon objected to simply stipulating (3). They wanted Bohmian mechanics to work with any initial probability distribution; that is, they wanted (3) more or less derived from (1) and (2). Bohm in fact never simply stipulated (3): Bohm 1952 (II.7) mentioned that the effects of collisions and other random processes would be to cause any differences between $\rho(x)$ and $|\psi|^{2}$ to decay with time, and in 1953 he wrote a paper directly on this topic. ${ }^{5}$ Neither result succeeds in rigorously showing that any initial probability distribution will work. As a result, for more than fifty years the distribution postulate has been the subject of scrutiny, with many Bohmians attempting to show that the postulate has essentially the same justification as the microcanonical probability measure does in statistical mechanics.

But what is the justification of the microcanonical probability measure in classical statistical mechanics? Few questions in all of the foundations of physics are more vexed and yield such a diversity of answers. There are Jaynesians, Boltzmannians, Gibbsians, Prigoginians, Krylovians, Khinchinians, interventionists, and more. Some believe the thermodynamic limit solves the foundational puzzles, others environmental perturbations, others symmetries and ignorance, others mixing dynamics, and others the H-theorem. ${ }^{6}$ The answers these theories give are sometimes no more similar than chalk and cheese. Faced with this foundational bedlam, we see that criticisms like Pauli's and Keller's are problematic: one certainly cannot uncontroversially do for the microcanonical probability distribution what they want done for (3), yet presumably neither they nor their advocates recommend the rejection of classical statistical mechanics. Today most Bohmians agree

\footnotetext{
${ }^{5}$ Keller's 1953 paper is odd, and my description misrepresents it slightly. The paper argues that unless (3) can be deduced from (1) and (2), then it is not "an ordinary statistical mechanics" of a deterministic theory-and this is supposed to be a bad thing. What is odd is that standard equilibrium statistical mechanics theory is not "an ordinary statistical mechanics" in the sense Keller means, as he acknowledges (fn 4). Keller means an "ordinary" statistical mechanics to be one where probability enters through ignorance. Even stranger is the fact that Keller cites but does not discuss the fact that twice Bohm tries in print to show (3) is an "ordinary statistical mechanics" in precisely Keller's sense, as Bohm tries to derive (3) from ignorance over environmental perturbations. So Bohm's theory is criticized for not living up to a standard that equilibrium theory doesn't live up to and attempts to live up to this standard are ignored.

${ }^{6}$ See Sklar 1995 for a good map through this literature.
} 
that the distribution postulate has roughly the same justification as the microcanonical probability posit — so long as no one asks what that justification is! If one asks this question, foundational bedlam arises again, this time at the sub-quantum level.

Before delving into the topic further, let's step back and ask why the distribution postulate is so needy of justification? Why do so many feel that (3) shouldn't stand alongside (1) and (2) as "axioms" of the theory? Why can't the theory posit three axioms? Let's call the position that treats (1)-(3) on a par as axioms of the theory the Default Position. According to it, we wouldn't ask, "why does the distribution postulate hold?" any more than we would ask why the Schrödinger equation holds. From the Bohmian perspective, there is as much evidence for (3) as for (1) and (2). So why do few hold this position? ${ }^{7}$

I don't believe that there are any knockdown arguments against the Default Position. But there are a number of considerations that, on balance, lead most to want to do better. The most obvious and easily justifiable rationale for distinguishing (3) arises from parsimony. Two axioms are fewer than three. So if one thought (3) were derivable or made plausible from the others, then that is better than merely positing (3). The more one can explain with less, the better. And since there is little prospect of deriving either (1) or (2) from the rest, (3) is a natural target. (Note, however, that Nelson does try to derive (1) from an equation very much like (2).)

Some may also feel a philosophical motivation for justifying (3). There is a widespread intuition that real laws of nature are dynamical. (1) and (2) and Hamilton's equations are dynamical, whereas (3) and the microcanonical probability measure are not. Real laws govern particles, fields, etc., and governing is something that happens in time. Dynamical laws are first-class citizens of the theory, whereas non-dynamical posits are second-class. Second-class citizens don't have the right to delimit what is physically possible. Hence Bohmian worlds in which $\rho=|\psi|^{3}, \rho=|\psi|^{4}$, and so on, are perfectly possible, as are anti-thermodynamic and a-thermodynamic worlds in the thermal case. Given the real laws, the feeling goes, we are lucky to inhabit a world with such striking generalizations as provided by thermodynamics and quantum mechanics. From this perspective, we are making something stable (thermodynamics, quantum mechanics) very contingent, and simply assuming the right probability distribution then seems ad hoc. Of course, one can challenge this rationale. Physics seems to posit plenty of nondynamical laws, e.g., the Pauli exclusion principle. And from the perspective of some accounts of laws of nature, the idea that laws must govern temporal evolution is unnatural. If a statement is simple and strong enough, that may qualify it as a law of nature. Or maybe the fact that Born's rule seems projectable warrants thinking of (3) as

\footnotetext{
${ }^{7}$ Bricmont 1991 ends his paper recommending that we treat (3) as an axiom. DGZ 1992 consider this question in section 4.
} 
lawlike. In any case, there are certainly plenty of perspectives on laws of nature that would permit elevating (3) to first class citizenship. ${ }^{8}$

Some may think that the nature of (3) warrants special justification. On one side of (3) we have what is supposed to be a real field in the world and on the other side we have a probability distribution. Intuitively, (3) links very different sorts of entities, it seems, no matter how one interprets probability. But again, we can imagine responses. What is it for one entity to be 'a very different kind' than another? Aren't there plenty of equations in physics linking quite different objects? And some Bohmians don't think of $\psi$ as a real field - at least not in the same sense as the electromagnetic field. The link is then between two objects, neither of whom is clearly understood. So it seems a bit premature to insist these sorts of entities, whatever they are, require special justification when linked.

Finally, (3), especially when properly understood in terms of an effective wavefunction, appears to demand an extraordinarily special initial condition of the universe. The initial configuration of the universe would have to be one that leads to (3) when measurement conditions obtain. But again, absent a metric over how special by contrast the laws (1) and (2) are, it's hard to make this intuition precise. It's also hard to make the special initial condition charge stick to (3) for many reasons (see Callender 2004).

The Default Position seems defensible. However, the desire for parsimony plus a number of more intuitive worries about (3) make (3) the natural candidate for explanation over (1) and (2). The question then is, can we do better than the Default Position?

This question immediately leads to another: what would "doing better" be? We can imagine a range of possible relationships between (3) and (1)\&(2). The weakest would be pointing out that there exists one initial condition such that it leads to the Born frequencies for subsystems of the universe. What would be wrong with this?

Intuitively, such a demonstration is not enough — not, as Dürr 2001 (p. 128) says, if we want to explain why (3) holds. We live in a world wherein the Chicago Bulls won three basketball championships in a row. Yet mechanics has sets of solutions wherein they never won and never even existed. Here mother intuition does not cry out for a special sort of explanation. The universe began in a state such that subsequent evolution takes us to a Bulls "three-peat", and that is that. Boston Celtics fans can bemoan their fate at living in such a universe, but most would not also complain that physics has sold us short explanatorily. Yet people would complain if Bohmian mechanics (classical mechanics) explained the quantum (thermodynamic) regularities the way we just explained the Bulls' feat. The difference stems from the fact that the regularities of thermodynamics and quantum mechanics form two of the most striking and pervasive patterns in our universe. To chalk them entirely up to the "chance" first microstate of the universe flies in the face

\footnotetext{
${ }^{8}$ For example, consider the so-called "Mill-Ramsey-Lewis" account of laws of nature. According to this theory, the laws are the axioms of that deductive system which is, on balance, simplest and most informative (see Lewis 1994).
} 
of the fact that we typically expect thermodynamic and quantum behavior. Physics seems like it is shirking its duty if it merely proclaims that there is an initial condition whose evolution leads to what you will see.

At the opposite extreme, suppose that we could show that for every initial condition the distribution given by (3) held after a certain length of time. (3) would be a deductive corollary of (1) and (2). Clearly this situation would be optimal. Its only downside is that it is provably impossible. There are plenty of otherwise perfectly acceptable wavefunctions that are not equilibrium wavefunctions. Real wavefunctions, for whom the Bohm dynamics predicts no motion, are one obvious example. (Furthermore, as we'll see, it's also provably impossible to show that (1) and (2) deductively entail distributions observationally indistinguishable from (3).)

That leaves us with the possibility that some, or better, most initial conditions yield (3) for subsystems. If (3) holds for most ways the universe could have been, the mystery over its occurrence is lessened. It is to attempts at showing this that we now turn.

\section{Dynamical Approaches I}

The guiding thought behind this approach is that the universe might have begun in quantum nonequilibrium and yet nevertheless evolved into quantum equilibrium. The Bohmian dynamics is supposed to "force" nonequilibrium initial states into equilibrium. Most of the work done on this approach predates the distinction between universal and effective wavefunctions; for the moment, therefore, let us pretend the distribution postulate (3) refers to the universal wavefunction, not the effective wavefunction. So understood the desired transition is, strictly speaking, impossible. The conservation equation (4) implies that a distribution in nonequilibrium can never get into equilibrium. That does not mean, however, that initial distributions such that $\rho \neq|\Psi|^{2}$ will not in time approach a distribution observationally indistinguishable from $\rho=|\Psi|^{2}$. It is compatible with the dynamics that various distributions initially such that $\rho \neq|\Psi|^{2}$ nonetheless evolve into $\rho=|\Psi|^{2} \pm \varepsilon$, where $\varepsilon$ is within the range of experimental/observational error. Given a suitable coarse-graining of $\rho, \rho$ may "look" like it is in equilibrium for all intents and purposes. The idea should be familiar from Gibbs: the Gibbs fine-grained entropy is stationary but the coarse-grained entropy can evolve with time.

This dynamical approach began with an early paper by Bohm. Bohm 1953 showed that an ensemble of two-level molecules out of equilibrium, when subjected to random perturbations, would approach the equilibrium distribution. Potel et al 2002 do something similar. Neither gives a general argument, and it is not clear how to interpret these random perturbations (see section 6). But others - most prominently Antony Valentini - have pursued this line of inquiry in more detail without adding random perturbations. Valentini argues that a coarse-grained distribution $\overline{\rho_{0}}$ of configuration variables will relax to coarse-grained equilibrium $\overline{|\Psi(q)|^{2}} \cdot{ }^{9}$. Recall Gibbs famous "ink

\footnotetext{
${ }^{9}$ See Valentini 1991 for precise definitions of the coarse-grainings.
} 
drop" analogy: when the ink and water are stirred, after a time the system will look homogeneous from a coarse-grained perspective. Here now $\rho$ and $\Psi$ play the role of ink and water, and the hope is that for complicated systems the dynamics will mix the two together so that they tend to become indistinguishable at a certain level. If this can be shown, the claim is that we wouldn't need to suppose (3) independently; rather coarsegrained equilibrium would hold now even if the universe didn't begin in equilibrium.

What can be shown? Valentini 1991 and then Valentini and Westman 2004 do essentially two things. First, Valentini shows that an "H-theorem" holds for Bohmian mechanics. The H-theorem is the Bohmian counterpart of Gibbs-Tolman's famous coarse-grained H-theorem. Defining a "sub-quantum entropy" $S$ in terms of a coarsegrained distribution, he argues that $S$ will attain its maximum value over time. Second, Valentini and Westman show how simulations of some nonequilibrium systems evolve to coarse-grained equilibrium. For these systems they also improve on the H-theorem by obtaining some estimates for the time it takes for relaxation to equilibrium to occur.

How should we understand these results? Valentini and Westman write:

"We interpret these results as further evidence that, in the pilot-wave formulation of quantum theory, the Born distribution $\rho=|\psi|^{2}$ should not be regarded as an axiom. Rather, it should indeed be seen as dynamically generated, in the same sense that one usually regards thermal equilibrium as arising from a process of relaxation based on some underlying dynamics." (3)

As noted above, one can agree that quantum equilibrium gets justified roughly the same way as thermal equilibrium, but disagree over what that justification is.

There are some reasons to be dissatisfied with Valentini's H-theorem. Let me mention two that also arise with Tolman's original coarse-grained H-theorem, and then one more specifically Bohmian worry. First, one must assume that the initial coarse-grained distribution $\overline{\rho_{0}}$ is equal to the initial fine-grained distribution-what Valentini calls the condition of "no fine-grained microstructure." Should we accept this condition? Given the context, it's not clear that this is a terrific gain. Is it so obvious that assuming $\overline{\rho_{0}(x)}=\rho_{0}(x)$ is less objectionable than assuming (3)? To me it is not. In both cases we're assuming the early configuration distribution had a rather special profile.

Second, the proof shows that $S\left(\overline{\rho_{0}}\right) \leq S\left(\overline{\rho_{1}}\right)$ if $\overline{\rho_{0}(x)}=\rho_{0}(x)$, but from this it does not follow that $S\left(\overline{\rho_{1}}\right) \leq S\left(\overline{\rho_{2}}\right)$. Nothing follows about the relative values of two entropies if both are after the initial condition. To get $S\left(\overline{\rho_{1}}\right) \leq S\left(\overline{\rho_{2}}\right)$, we would then need to invoke $\overline{\rho_{1}(x)}=\rho_{1}(x)$. In effect, we need to rerandomize the dynamics. Perhaps there is way of looking at things whereby this rerandomization is caused by the measurement preparation. If not, and maybe even if so, the effective rerandomization needed looks suspect. If it's not clear that assuming $\overline{\rho_{0}(x)}=\rho_{0}(x)$ even one time is less objectionable than assuming $\rho=|\Psi|^{2}$, it's even less clear that assuming it over and over again is preferable. 
Third, as mentioned, this literature doesn't distinguish between effective and universal wavefunctions. To make sense of it I have interpreted it in terms of universal wavefunctions. But as stressed at the outset, what is really needed is a justification of (3), where (3) is the effective wavefunction. $\rho=|\Psi|^{2}$ is not sufficient for $\rho=|\psi|^{2}$ to hold in measurement situations. Even if one proves that the universe as a whole is in quantum equilibrium, we really want to prove that patterns inherent in subsystems of the universe are in quantum equilibrium. Whether any results mentioned survive the move to the proper understanding of (3) is not clear. The effective wavefunction is not always welldefined, and most of the results hang on $\psi$ always evolving according to the Schrödinger evolution. If no transference is possible, then results like Valentini's will have more relevance for cosmology than for justifying Born's rule.

What should we make of Valentini's demonstrations of quantum nonequilibrium states that evolve to coarse-grained quantum equilibrium? Here I believe it is useful to contrast a strong and a weak claim. Valentini seems to vacillate between the two. The strong claim is espoused in the quotation above. It claims one is giving a purely dynamical explanation of the distribution postulate. The weak claim, by contrast, is merely that (3) could be weakened, for there are nonequilibrium initial states that develop into states that might — for all we know - be the universe we're in. The weak claim, in other words, is that some nonequilibrium states do evolve into states that are observationally indistinguishable from equilibrium.

The strong claim is too strong. None of the results cited justify the strong claim. Valentini and Westman (section 6) acknowledge that one will never prove that there exists a monotonic approach to coarse-grained quantum equilibrium for all initial conditions. The reason for this is that Bohmian mechanics possesses a deterministic time-reversible dynamics. The time reversibility implies that there will be solutions of the dynamical equations that take a system away from equilibrium, rather than toward equilibrium. So if what makes the dynamical strategy "dynamical" is its ability to show that every set of initial conditions out of quantum equilibrium will eventually go to quantum equilibrium, then the above facts mean this desire will be frustrated.

The weak claim, however, is probably true and potentially interesting. Their examples do make plausible the assertion that some nonequilibrium systems will nonetheless look like ones in equilibrium for long times. The number of Bohmian initial conditions giving rise to a quantum universe is thus enlarged in some sense, alleviating some of our guilt over positing a special initial condition. However, nothing Valentini says shows that most or even $a$ lot of the initial states are such as to lead to quantum predictions. Inasmuch as that is what we wanted, we can keep the 'weak' version of his claim in mind and still demand more.

Finally, attention ought to be paid to the evidential disanalogy between the Gibbs-Tolman argument in thermodynamics and Valentini's for quantum mechanics: in the thermal case there is no question that we need to describe the non-equilibrium case-we're in it - but in the quantum case there is no evidence that we were ever in non-equilibrium. Valentini 
hopes that positing a universe in quantum non-equilibrium will turn out to be explanatorily useful in some way, e.g., in cosmology. Then we would have independent evidence that the world was once out of quantum equilibrium. Until then, however, the question of how we get to equilibrium from non-equilibrium is not thrust upon us with the same force as it is in the thermal case.

\section{Dynamical Approaches II}

Another type of dynamical approach mirrors the classical ergodic justification of the uniform measure in thermal equilibrium. Here the idea is not to show that quantum nonequilibrium states will inevitably approach equilibrium states. Rather, they are attempts to justify the $|\psi|^{2}$ density measure used in equilibrium, based on the alleged ergodic feature of the dynamics. The dynamics would then guarantee, for all but a set of measure zero, a quantum equilibrium distribution.

Classically, an ergodic system is one whose infinite time averages are equal to its phase averages, where the phase averages are calculated using the microcanonical measure. If one can argue that what we observe are roughly infinite time averages, then if the system is ergodic we can understand why using the microcanonical measure is justified. Physically, the idea is that ergodic systems spend time in a region of phase space equal to the proportion of that region compared to the full phase space, as measured by the microcanonical measure. Since most of the equilibrium phase space corresponds to a Maxwellian distribution, ergodic dynamics assures us that the distribution will be Maxwellian.

Transferred to the Bohmian case, the reasoning would be something like the following. Assuming a stationary wavefunction (see below), one would first show that the probability the configuration variable $\mathrm{x}=\left(\mathrm{x}_{1} \ldots \mathrm{x}_{\mathrm{n}}\right)$ was in region $\mathrm{W}$ is given by the infinite time limit of the ratio of time it spends in $\mathrm{W}$ over the time it spends in the total available space. An ergodic theorem would then link this infinite time average with the measure of the region $\mathrm{W}$ given relative to effective wavefunction $\psi$. This last function would be equal to $|\psi|^{2}$. So the ergodic theorem would show that the infinite time average is given by $|\psi|^{2}$. Hence, if one could argue that what we measure are essentially infinite time averages (a big if!), one would have a justification of the $|\psi|^{2}$ density.

What has been shown? Not much. Shtanov 1997 believes equilibrium is justified by ergodic behavior in the classical case and simply assumes/hopes that it holds in the Bohmian case. He then gives a more detailed sketch than above of how, if the Bohmian dynamics is ergodic, this might yield quantum equilibrium. Geiger, Obermair and Helm 2001 claim to have demonstrated that Bohmian dynamics is ergodic and derived quantum equilibrium from this ergodicty. Their proof is not as rigorous as one might expect. The claim is instead that given $|\psi|^{2}$, the series of measurements and re-preparations leads to a Baker's transformation of the available area in phase space, and that hence the trajectory of the measured system is ergodic. In many respects the argument is more similar to one by DGZ 1992 than the classical counterpart of ergodicity under consideration here; we'll discuss that more detailed program in section 7 . 
Since so little has been done we cannot say much about an ergodic justification of (3). Let me simply mention some distinctive issues that arise in the switch to Bohmian mechanics from classical mechanics. The dynamics is quite different from classical dynamics, so one can expect many additional complications. One general one that stands out is that the Bohmian dynamics is equivariant, not stationary, yet ergodic theory requires that the measure be invariant in time. Shtanov's sketch assumes that we're using a stationary wavefunction, but this assumption needs to be removed. Another is that our statement needs to be in terms of the effective wavefunction, not universal wavefunction, if it is to be relevant to Born's rule; yet classical ergodic theory does not have such a distinction. Another more philosophical distinction is that one doesn't have the problem of non-equilibrium phenomena in the Bohmian case. Non-equilibrium phenomnena, observed in the classical case, sit awkwardly with the ergodic justification. If measurements reveal infinite time averages and these are always the same, how can we ever see non-equilibrium phenomena--characterized by having different values at different times? Since we haven't ever observed quantum non-equilibrium, perhaps the Bohmian ergodicist is not as hard pressed by this objection. That said, the Bohmian ergodic approach clearly will inherit many of the problems one finds in the thermal case, and these are considerable. In particular, one wants a rationale for identifying infinite time averages with what we meet in finite observation times and some assurance that realistic systems truly are ergodic (see Earman and Redei 1996).

\section{New Dynamics.}

This strategy exchanges one fundamental dynamics for another. The hope is that a new "improved" dynamics is amenable to a dynamical justification of (3) where the old dynamics given by (1) was not. In the classical case, it means jettisoning Hamiltonian dynamics for something else. This something else might be, for example, modifying Hamiltonian equations with a stochastic kick term added to the Liouville equation. With such a modification it may be possible to deduce likely convergence to quantum equilibrium. $^{10}$

Immediately we should pause to point out that if one treats this stochastic kick term as merely an epistemic convenience, reflecting our ignorance over environmental perturbations, then this move doesn't really answer our question. It merely pushes it back a step, for we would be entitled to ask why one chose the probability distribution one did over the perturbations. Assuming some natural probability distribution over the environment is perfectly fine for practicing physics. But if one wants to get at the ultimate reason for quantum equilibrium, it is merely obscurantism. To fully pursue this avenue, one needs to rewrite the fundamental equations.

This more drastic strategy is much more popular among Bohmians than the corresponding strategy is in classical statistical mechanics. The reason for this difference

\footnotetext{
${ }^{10}$ See van Lith section 14.2 for discussion of two ways of accomplishing this in the
} thermal case. 
is not surprising. Because we only see the quantum distribution, any dynamics that reproduces quantum equilibrium will be empirically adequate. And since we don't have independent observable evidence that trajectories obey (1), researchers no doubt feel free to alter the form of (1) in a way they would not feel free, classically, to alter the form of Hamilton's equations. In classical mechanics, Hamilton's equations are vindicated daily via our observations of satellites, balls, etc., and the Maxwell distribution underlying equilibrium is the more hypothetical state. In Bohmian mechanics the evidence is reversed: quantum equilibrium is vindicated daily in labs around the world, whereas the guidance equation is the more hypothetical object. Not for a minute do I mean to suggest that there is little evidence for (1); I'm merely pointing out an observational asymmetry. The success of the whole Bohmian package helps confirm (1).

For our purposes, modifications of the guidance equation (1) are important for two reasons. First, the modifications may have repercussions for the justification of the distribution postulate. Indeed, many of the modifications offered in the literature, including the first one by Bohm and Vigier, were intended as answers to this problem. By modifying (1), one hopes that the dynamics will show improved mixing behavior, taking the nonequilibrium system quickly to equilibrium. Second, many modifications of (1) - done to justify the distribution postulate or not - make the dynamics fundamentally stochastic. Bohmian mechanics is indeterministic according to these modifications, and inasmuch as this indeterminism may affect the interpretation of quantum probabilities, we need to pay attention.

How can one modify (1) and maintain empirical adequacy? As mentioned, so long as quantum equilibrium is maintained, any theory may be empirically adequate. It should not be at all surprising that there are other possible trajectories that give the same distribution. (1) is attractive because it is very simple, natural and possesses various important symmetries, but it is not the only one. (Notice that the same can be said of the classical Hamiltonian equations too. This is a more or less general feature of science, not something specific to Bohm.) As Deotto and Ghirardi observe, it is obvious that we can add a velocity field to (2) and still preserve our continuity equation if the field has the form

$$
v_{o}=\frac{j_{o}}{|\psi|^{2}}
$$

and $\nabla \cdot j_{o}=0$. Deotto and Ghirardi give examples of alternative deterministic guidance equations. But there are also many stochastic variations. These come in essentially two kinds: the first is stochastic because stochastic terms have been added to (1), whereas the second is stochastic because the fundamental quantities - in Bell's terminology, beables - are assumed to be discrete and bounded.

Regarding the first, Bohm and Vigier 1954 introduced "fluid fluctuations" that they hoped would drive an arbitrary system to quantum equilibrium. Bohm and Hiley 1993 and Peruzzi and Rimini 2000 have done something similar. Also, if one ignores Nelson's attempt to derive the Schrödinger equation from his stochastic guidance equation, one 
can think of his stochastic mechanics as a Bohmian theory where (1) is replaced by a stochastic guidance equation. Indeed, there is a whole family of stochastic guidance equations that preserve quantum equilibrium:

$$
\frac{d x}{d t}=b d t+\sqrt{\alpha} d \omega
$$

where $b=\frac{\hbar}{m} \nabla S+\alpha \frac{\hbar}{2 m} \frac{\nabla|\psi|^{2}}{|\psi|^{2}}$ and $\mathrm{d} \omega$ is a Wiener process, the simplest continuous

Markov process. If $\alpha=1$, then we get Nelson's mechanics and if $\alpha=0$ we get Bohmian mechanics, i.e., equation (1). See Davidson 1979 and Bacciagaluppi, forthcoming, for discussion of these and other related theories.

The second approach does not add a stochastic term to (1). Rather, it is an extension of Bohmian mechanics to discrete beables. Indeterminism results because trajectories for time-dependent discrete quantities cannot be continuous, so a differential equation of motion cannot be used. A stochastic dynamics is thus natural for discrete beables. Bell devised such a dynamics for fermion number density, and Bub, Vink and DGZT 2005 have developed this approach in more detail. ${ }^{11}$ The idea is as follows. We keep the wavefunction evolving according to the normal deterministic Schrödinger equation. As in Bohmian mechanics, we then introduce a position-like beable, which we'll call $o$. The observable Ô corresponding to $o$ has finite and discrete eigenvalues $o_{n}$. What replaces (1) is now a transition matrix $T$. The transition matrix $T_{m n} d t$ gives the probability $o_{m}$ will jump to $o_{n}$ in time $d t$. Just like the ordinary Bohmian velocity, the transition matrix is equal to the probability current $J$ divided by the probability density $\mathrm{P}_{\mathrm{m}}$, only here these two are discretized (for details, see Vink). Thus:

$$
T_{m n}=\frac{J_{m n}}{\hbar P_{m}}
$$

so long as $\mathrm{J}_{\mathrm{nm}} \geq 0$. $\mathrm{T}_{\mathrm{nm}}=0$ if $\mathrm{J}_{\mathrm{nm}} \leq 0$. This dynamics describes a biased random walk in Ô-space. Vink proves that in the continuous position limit, this stochastic dynamics converges on the original Bohmian dynamics. As in the deterministic case, one can add Deotto-Ghirardi currents for alternative empirically adequate trajectories; one also has some extra freedom in the choice of $\mathrm{T}_{\mathrm{nm}}$ (see Bacciagaluppi 10, Vink 48), thereby increasing in some sense the number of possible dynamics preserving quantum equilibrium.

Apart from introducing indeterminism, do the above theories add anything new to the discussion of the distribution postulate? Yes and no. Clearly the stated goal of those

${ }^{11}$ For the Bohmian, as opposed to Vink and Bub, the primary motivation for such a change has been the search for beables appropriate for quantum field theory (as opposed to Vink and Bub's desire to maximize the number of determinate observables together consistent at one time). 
adding stochastic additions to (2) is often to provide a "dynamical" explanation of quantum equilibrium, in the sense of section 4 above. Some have also expressed the claim that the Bell-Vink dynamics makes a difference here. Intuitively, one can see that adding extra fields to the velocity formula might induce more "mixing-like" behavior than already found in (2). But what has actually been shown? To my knowledge, the situation is not much different than the one surveyed above in 4 . There are scattered results: showing convergence to approximate quantum equilibrium with certain dynamics and particular systems, showing lack of convergence for other systems. Certainly there is nothing like a proof that for all or even most initial conditions the approach to equilibrium is inevitable or even probable. Nor, I speculate, is it likely that there will be such a dynamics and corresponding proof in the future. If this is so, we can repeat the claims and discussion from section 4 here. If we're looking for the impossible, i.e., showing that any probability distribution over initial conditions will lead to quantum equilibrium, then - surprise! — we do not find the impossible. We do, however, find nonequilibrium states that will evolve to worlds that are observationally indistinguishable from ours, but we do not have any reason to think most non-equilibrium worlds are like this.

\section{Typicality}

So far "dynamical" approaches have failed to show that "most" Bohmian initial conditions lead to a quantum world. And most have targeted the universal wavefunction instead of the effective wavefunction. Let us now turn to a strategy inspired by Maxwell and Boltzmann (like so many of the others) that explicitly formulates what we mean by "most" and then demonstrates that most initial conditions lead to Bohmian histories wherein Born's rule (for effective wavefunctions) is appropriate. In thermal physics, Maxwell famously showed that of all equilibrium empirical distributions of velocities, most are Maxwellian. And in non-equilibrium cases, Boltzmann made plausible the claim that, given an initial low entropy macrostate, most subsequent trajectories head to higher entropy states for long periods. "Most" in both cases is understood with respect to Lebesque measure restricted to a constant energy surface. DGZ do something similar for Bohmian mechanics. As with Maxwell and Boltzmann's demonstrations, the DGZ argument still leaves open many philosophical questions about probability.

The DGZ position is subtle, and they claim that various commentators haven't described their position accurately, so it behooves us to pay careful attention to the argument. The main argument is laid out extensively in DGZ 1992, although they comment and elaborate on the position in subsequent papers and lectures. What they essentially prove in 1992, as Dürr 2001 (p. 128) says, is a law of large numbers theorem for the empirical distribution of configuration values for subsystems of the universe. To get a feel for what they do, they provide a useful analogy:

Roughly speaking, what we wish to establish is analogous to the assertion, following from the law of large numbers, that the relative frequency of appearance of any particular digit in the decimal expansion of a typical number in the interval $[0,1]$ is $1 / 10$. In this statement two related notions appear: typicality, referring to an 
a priori measure, here the Lebesque measure, and relative frequency, referring to structural patterns in an individual object. (1996,38).

Understanding the example will help our cause. In mathematics, simply normal or just normal numbers are typical. If we take a number $\mathrm{x}=\mathrm{y} \cdot \mathrm{x}_{1} \mathrm{x}_{2} \mathrm{x}_{3} \ldots$ then $\mathrm{x}$ is normal just in case, for all bases and for all subscripts $i, 1 / 10^{\text {th }}$ of the $x_{i}$ 's are zero, $1 / 10^{\text {th }}$ of the $x_{i}$ 's are one, $1 / 10^{\text {th }}$ of the $x_{i}$ 's two, and so on. There are two remarkable facts about normal numbers. One is that we know of only one non-trivial normal number, a number discovered in 1933 by an undergraduate student named David Gowen Champernowne. (The number is $0.1234567891011 \ldots$ ) It is still unresolved whether many famous and important numbers, such as pi, are normal. Pi looks like it is normal, in the sense that its known relative frequencies are converging towards normalcy. ${ }^{12}$ But as we know, the finite relative frequencies are compatible with any infinite relative frequencies. What really suggests pi's normality, and every other number's normality, is the second remarkable fact: Borel showed in 1909 that "almost all" numbers x are normal. That is, Borel proved that the complement of the collection of normal numbers in $[0,1]$ is of zero length according to Lebesque measure. It is in this sense that normal numbers are typical.

Borel's theorem, it turns out, is a consequence of the strong law of large numbers (LLN) in probability theory. The strong law of large numbers says, roughly, that if $X_{1}, X_{2}, X_{3}, \ldots$ is an infinite sequence of random variables that are independent and identically distributed, then the relative frequency of some property converges almost surely (i.e., with probability one) to the probability of that variable. The weak law says, by contrast, that the convergence happens probabilistically, i.e., with probability strictly less than one. (One can also prove LLN-type results without assuming independence, but rather by showing the sequence is ergodic.) In the case at hand, the probability measure gives each numeral, ' 1 ', ' 2 ', etc., an equal probability of $1 / 10$. Furthermore, the numbers are identically distributed and independent: for instance, knowing $\mathrm{x}_{2}=4$ doesn't affect the probability of (say) $x_{3}=1$. Applied to the case at hand, the LLN states that if we pick a number uniformly at random between zero and one, then it is normal with probability one.

Notice that the decimal expansion of a number is "deterministic." There is nothing chancy about whether the next number of pi after 3.141 is 5 or not. Notice also that normality is a feature of the entire number, not any particular $\mathrm{x}_{\mathrm{i}}$. We don't say having a ' 5 ' in the sixth decimal location is normal. Normality is a feature of a collective of numbers having a certain pattern.

12 The frequency distribution for pi for the first 6,000,000,000 decimal places is (Kanada 1995):

'0': 599963005; '1': 600033260; '2': 599999169; '3': 600000243; '4': 599957439;

'5': 600017176; '6': 600016588; '7': 600009044; '8': 599987038; '9': 600017038; 
Return to Bohmian mechanics and Born's rule. Again we have a deterministic theory, and the counterpart of our numbers $x$ are histories of the Bohm particles $Q(t)$. As we found patterns in the x's, we try to find patterns in the configuration variable histories. In particular, the pattern we are interested in is the one wherein the particles for subsystems of the universe are distributed according to $|\psi|^{2}$ when the subsystem merits an effective wavefunction, just as we might look for the relative frequency of fives in a real number $\mathrm{x}$. The a priori measure, the counterpart to Lebesque measure, is the natural volume measure on configuration space, modified by $\left|\Psi\left(\mathrm{q}_{1} \ldots \mathrm{q}_{\mathrm{N}} ; 0\right)\right|^{2}$ :

$$
\left|\Psi\left(q_{1} \ldots q_{N} ; 0\right)\right|^{2} d^{3 N} q
$$

Let me note immediately that (6) uses the wavefunction of the universe, but what we are interested in is subsystems of the universe.

To prove a LLN result, one needs independent and identically distributed random variables. In the case of Bohmian mechanics, it is hardly obvious that one can get this for configurations of sub-systems of the universe, e.g., systems in the lab. After all, one would naively expect that if we take one subsystem $X$ at time $t$, evolve it to time $t$ ', where $\mathrm{t}^{\prime}>\mathrm{t}$, then $\mathrm{X}(\mathrm{t})$ and $\mathrm{X}\left(\mathrm{t}^{\prime}\right)$ are not independent. In fact, as DGZ point out, if the wavefunction is the ground state, not only is $X\left(t^{\prime}\right)$ a function of $X(t)$, but $X(t)=X\left(t^{\prime}\right)$, which is as far from independence as it gets. Nevertheless, under the conditions when an effective wavefunction is defined, matters are quite different. In this case one can show that if we have a collection of sub-systems $\left\{\mathrm{X}_{1} \ldots \mathrm{X}_{\mathrm{n}}\right\}$, either at a time or across time, each with the same effective wavefunction, then one can prove that the configurations of these sub-systems are in fact independent and identically distributed random variables with respect to the universal measure (conditioned on the environment of the subsystems). That is, the sub-system particle distribution, when effective wavefunctions obtain, is randomly and independently distributed according to $|\psi|^{2}$, both at a time and at different times. See DGZ for the precise argument. This is an important result, one making agreement with the quantum formalism possible. What DGZ have done, in effect, is argue that a kind of "effective Bernoulliness, and hence an effective ergodicity" holds in Bohmian dynamics (DGZ, 1992, p. x). When systems are large enough, the positions of Bohm particles in the measured subsystems "forget" the initial distribution in a sense.

With this independence result in hand, DGZ are then able to prove a LLN result. DGTZ (ms) summarize their work as follows:

In Bohmian mechanics, a property $\mathrm{P}$ is typical if it holds true for the overwhelming majority of histories $\mathrm{Q}(\mathrm{t})$ of a Bohmian universe. More precisely, suppose that $\Psi_{\mathrm{t}}$ is the wave function of a universe governed by Bohmian mechanics; a property $\mathrm{P}$, which a solution $\mathrm{Q}(\mathrm{t})$ of the guiding equation for the entire universe can have or not have, is called typical if the set $\mathrm{S}_{0}(\mathrm{P})$ of all initial configurations $\mathrm{Q}(0)$ leading to a history $\mathrm{Q}(\mathrm{t})$ with the property $\mathrm{P}$ has size very close to one, $\mathrm{S}_{0}(\mathrm{P})\left|\Psi_{0}(\mathrm{q})\right|^{2} \mathrm{dq}=1-\varepsilon, 0 \leq \varepsilon 1$, with "size" understood relative to the $\left|\Psi_{0}\right|^{2}$ distribution on the configuration space of the universe. For instance, 
think of $\mathrm{P}$ as the property that a particular sequence of experiments yields results that look random (accepted by a suitable statistical test), governed by the appropriate quantum distribution. One can show, using the law of large numbers, that $\mathrm{P}$ is a typical property.

With size understood relative to (6), DGZ show that a certain property $\mathrm{P}$ of solutions $\mathrm{Q}(\mathrm{t})$ are typical. The property $\mathrm{P}$ is essentially a pattern in $\mathrm{Q}(\mathrm{t})$, just as real numbers might display a pattern such as manifesting equal frequencies of numerals. Here the pattern is more complicated: it is having a configuration such that, when subsystems of it merit effective wavefunctions, the actual subensembles have probability density of positions given by $|\psi|^{2}$. This pattern is precisely what needs to be the case for Born's rule to be appropriate. The claim that $\mathrm{P}$ is typical is another way of saying that a LLN result has been proven such that most (with respect to measure (6)) Bohmian histories $\mathrm{Q}(\mathrm{t}$ ) have property $\mathrm{P}$. We finally have a proof that most Bohmian universes are such that Born's rule works in them.

Does the proof beg the question? Though we haven't gone through all the mathematical details, the worry is obvious: one assumes a measure weighted by the quantum state squared to show that $|\psi|^{2}$ behavior is typical. Now, strictly speaking, no one is assuming what is to be proved. The assumption is at the level of $|\Psi|^{2}$ not $|\psi|^{2}$. And one is not assuming that there is an empirical distribution in accord with $|\Psi|^{2}$. It is not like one has an ensemble of 100 people, $90 \%$ of whom have black hair, and then (randomly) extracts a sub-ensemble of 50 and finds that roughly $90 \%$ of these too have black hair. The connection between $|\Psi|^{2}$ and $|\psi|^{2}$ is more complicated than that. That said, the objector might respond: true enough, but still, if one assumed the volume measure was weighted by $|\Psi|^{3}$ then it would show that some other behavior was typical, or no behavior at all. Granted, $|\Psi|^{2}$ is at the level of the universe and $|\psi|^{2}$ is at the level of individual effective wavefunctions, so the proof is not trivial. But still, the objection goes, why choose $|\Psi|^{2}$ rather than $|\Psi|^{3}$ ? Or why choose $|\Psi|^{2}$ rather than the uniform measure? As Bricmont 2001 remarks, $|\Psi|^{2}$ hardly seems the a priori natural choice if, say, the universe were just a particle in a box.

In response, let me first note that we have to choose a measure. If the objection is to imposing a measure at all then this style of explanation cannot get off the ground. The objector is then in the awkward situation of denying the explanatory value of some of the prima facie most elegant explanations in science. Second, if the objection is that any choice of measure that works is ad hoc, then so long as we're careful to not define ad hocness in terms of the measure working, DGZ are not in trouble. There are various mathematico-physical facts that single out (6). For instance, DGZ respond by pointing out that $|\Psi|^{2}$ is an equivariant measure. A non-equivariant measure will therefore pick out a time as special. Why pick a measure that arbitrarily distinguishes one time over others? If we chose $|\Psi|^{3}$, and then backward evolved it to the early universe, the measure would no longer be $|\Psi|^{3}$. $|\Psi|^{2}$, by contrast, is time translation invariant. To this I would also add that (6) is the measure used to demonstrate global existence and uniqueness of the dynamics. The results on global existence and uniqueness prove that almost all trajectories as measured by (6) don't run into nodes of the wavefunction, don't escape to 
infinity, and don't run into singularities of the potential. Dialectically, we're assuming already that (1) plus (2) give us a workable global dynamics and this is a precondition for even worrying about the status of (3). So in a sense, (6) is already part of the Bohmian "package" and distinguished this way too.

Finally, as Maudlin (ms) emphasizes in discussing a lecture of Dürr, to some extent the charge "why that measure?" can be alleviated by showing that measures besides (6) will also work. In the case where we have an infinite number of random events, then so long as that measure is absolutely continuous with (6) the LLN result will hold good for it too. This is a point familiar from ergodic theory. A measure absolutely continuous with (6) agrees with (6), by definition, on the size of measure one and measure zero subsets.

Since (6) assigns measure one to those histories in which Born's rule is appropriate, any measure a.e. with respect to (6) will too. In the more realistic finite case, we also have some freedom in what measure we pick, so long as we don't pick a measure that weighs heavily the formerly very tiny sets of positive measure. See Maudlin for more on this point.

I do not, therefore, have much sympathy with the claim that (6) begs the question. If a question is being begged, I'm not sure that anyone can beg less and still end up claiming "most" of anything evinces any kind of pattern. One could, I suppose, reply so much the worse for saying most of any real-numbered valued quantity display any property. My reply is that the cost of maintaining this position is unacceptably high. Some of the most elegant and powerful explanations in science are of this form, e.g., Maxwell's treatment of equilibrium, Boltzmann's treatment of non-equilibrium.

To acknowledge that DGZ provide a structurally similar explanation of Born's rule to explanations by Boltzmann and Maxwell is not to say we fully understand any of these explanations. As things now stand, we have a LLN result. That is it. It is a piece of mathematics. How does this result relate to actual frequencies? How does it relate to rational credence? These and other questions need answers.

\section{Interpretation of Quantum Probability}

We are now ready to discuss the interpretation of quantum probabilities in a Bohmian world. Let me begin with three general points. First, when speaking of probabilities in a deterministic theory one often hears it said that the probabilities are "epistemic" or "ignorance" probabilities. Usually by ignorance one means that the probabilities are interpreted as one's subjective degrees of belief in a proposition. Certainly this interpretation makes it easy to square non-trivial probabilities with a deterministic context. Thanks to ignorance one's credence in a proposition need not be one or zero. But one should not be satisfied with a subjective interpretation. It's not just that $I$ think it's likely that the system I'm measuring is in quantum equilibrium - though if I do that's a good thing because it's true. It's likely to be in quantum equilibrium regardless of what I or anyone else believes. And our thinking or believing it isn't responsible for Born's rule being essentially lawlike in a Bohmian universe. That would endow we human beings with far too much power. There can be probabilistic patterns even in a deterministic 
universe, and (3) describes one of them; nothing about my beliefs explains why this pattern holds. I do not have space to launch a developed criticism of subjectivist interpretations of quantum probability, but I hope that I have said enough to at least motivate the search for a non-subjective interpretation of these probabilities.

Second, I don't believe that much of what follows hangs on the fine details of Bohmian mechanics. I doubt that the interpretation of objective probability in science varies greatly between scientific theories, never mind interpretations of quantum mechanics. There are three reasons for my skepticism. First, there aren't a great many even remotely satisfactory theories of objective probability to spread around. For example, one shouldn't say Popperian propensities find a natural home in a GRW world, frequentism in a Bohmian world, and subjectivism in an Everettian world. The reason for this is that one shouldn't believe in Popperian chances or the others in any world! The reader can find the usual litany of objections against these theories elsewhere (e.g., Hajek) and I shall not repeat them. Second, the best interpretation of objective probabilities, when it emerges, will probably be best in virtually all theories. Maxwell's equilibrium theory, Boltzmann's theory, games of chance, fitness in biology, and so on, all require objective probabilities to play very similar roles, as one sees in Strevens 2003. Third, although the difference between an underlying deterministic dynamics and indeterministic dynamics might be significant, even here the difference may be over-stated. As Saunders 2005 remarks, at the mathematical level the only difference between deterministic and indeterministic dynamics is where one puts the measure: in the deterministic case, the measure is defined over the space of Cauchy data at one time, in the stochastic case it is defined over the space of entire histories. While this difference can matter, I'm not sure that it should or does according to the best interpretations of probability.

The third general point I want to make is simply to emphasize the obvious: one cannot "read off" an interpretation of probability from a mathematical/physical result. The ergodic theorem, the LLN, and even indeterministic dynamics are compatible with a variety of interpretations - even if the reverse is sometimes not true. Reichenbach and others famously saw the ergodic theorem as the holy grail of the interpretation of objective probability; and more recently, von Plato asserts that it justifies a "time average" interpretation of probability — not in general, but for systems that are ergodic. One can see the appeal: the equivalence of phase space averages and time averages in an ergodic system suggests interpreting the $\mathrm{P}(\mathrm{A})=0.5$ as simply stating that the system spends half its time in state A. As we know, this interpretation has its problems; but even if it did not, the technical result is more plausibly a necessary rather than sufficient condition for the "time average" interpretation.

Other articles in this volume discuss interpretations available only to systems with stochastic dynamics, so I will not spend time on that question here. I will only make the obvious point that if Bohmian mechanics is stochastic, as it is with a variety of guidance equations, then many of the claims made in these papers apply also to Bohmian mechanics. Here I want to focus on the distinctive type of explanation offered by DGZ of the Bohmian probabilities. 
DGZ prove a LLN result for Bohmian mechanics. This brings their justification squarely in line with Maxwell's LLN result for Maxwellian velocities in equilibrium and Boltzmann's for the evolution to equilibrium. Having a Maxwell-Boltzmann-like explanation of (3) leaves most philosophical questions about this explanation, in particular, the interpretation of probabilities, as more or less open. DGZ do make various claims about how they don't want this result to be interpreted, but this is consistent with not adopting a particular theory.

We begin by showing that there is a need to interpret the probabilities in DGZ's result. As with the ergodic theorem, there is a temptation to view a LLN result as itself supplying an interpretation of probability. Many textbooks take the LLN to be implying a relative frequency interpretation of probability. ${ }^{13}$ Among workers in probability theory, even among those not identifying chance with relative frequency, there is also a tendency to view the LLN as the key in linking chances with relative frequencies. One devises a theory of chance and then shows its relationship to the empirical frequencies via the LLN (see Howson's 1995 discussion of Dawid and Mellor).

But this is a mistake. The LLN states that in the long run the relative frequency of an i.i.d. distributed random variable and the probability of the relevant outcome (derived from the assumed measure) are with probability approaching or equal to 1 nearly identical. Put loosely, probability is close to long run relative frequency, probably. Clearly, the second use of probability needs a non-probabilistic explication to avoid circularity (as does "independence" in iid). The LLN does not provide an interpretation of probability, nor does it even link long run frequencies to probabilities without an understanding of the second probability.

Another way to approach the question is to ask, what does typicality actually imply? Suppose that you prepare a quantum measurement. You wonder what distribution of particle positions you should expect upon measurement. DGZ come forward and say, "Use Born's rule - this is a great policy in typical Bohm histories." You think about it and ask the natural question, "why should I expect this universe to be typical? Is it likely that this universe is typical? Can't atypical things happen?"

Dürr 2001 answers:

Are typical events most likely to happen? No, they happen because they are typical. But are there also atypical events? Yes. They do not happen, because they are

${ }^{13}$ Some Bohmians sound as if they are using the LLN to provide a relative frequency interpretation, too, even if that is not their intention: "Using typicality one may define probability in terms of law of large numbers type statements, i.e., in terms of relative frequencies and empirical statistics. What is meant by: In a Stern-Gerlach experiment the probability for spin up is $1 / 2$ ? The same as for heads turning up in a coin tossing. So what is meant? The law of large numbers: That in a long run of repetitions of the experiment the relative frequency of the outcome in which the spin is up or the coin shows heads will typically be close to the value $1 / 2$ " (Dürr 2001, 130). 
unlikely? No, because they are atypical. But in principle they could happen? Yes. So why don't they happen then? Because they are not typical. p. 130.

Dürr notes that there is a great deal to say philosophically about this position. Indeed there is. As we have seen, we are here reprising a famous episode in the history of probability. In the history of probability, there have been many attempts to answer this problem. Cournot and others tried to answer the problem by stipulating that small probabilities are impossible, but this leads to serious consistency problems - Dürr wisely avoids this position in the quote. Kolmogorov instead said that measure one results implied "practical certainty," yet this position gives the probabilities a subjective cast, and so it won't meet our needs for an objective probability.

Note that this problem appears most conspicuously in the ergodic justification of statistical physics. This fact is not surprising, since the ergodic theorem can be read as a LLN result (in fact the Chebyshev inequality, used by Khinchin, is a LLN result saying that as $\mathrm{n}$ grows larger, the number of trajectories whose time-averages and spaceaverages differ observably grow rarer). The ergodic theorem claims that with measure equal to one, the time average probability and the statistical probability are identical. The famous "measure zero" problem for ergodic justifications asks why we should assume measure zero events are unlikely to happen. This is a particularly devastating problem for those scientists and philosophers who hoped that the ergodic theory provided its own interpretation of probability. But it is equally a problem for anyone pinning the interpretation of probability on the LLN.

Although DGZ shun the identification of typicality with likeliness, it is also clear from the Dürr quotation that they don't think one should bet much money on atypical phenomena occurring. Unless we solve the problem mentioned above and connect their result to empirical frequencies, then it's unclear why not and it's also unclear what the LLN result is doing for us. Like probability, typicality is a guide to our beliefs. It seems incumbent upon us to interpret this "second probability" in the LLN as a probability. The question then is what kind of probability is it?

DGZ express serious reluctance to the idea of interpreting the typicality measure probabilistically. We can understand this and sympathize by looking at their result in terms of the two most dominant interpretations of objective probability, frequentism and propensity theory. Suppose we interpret the second probability in the LLN result in terms of relative frequencies, either Reichenbachian ideal actual frequencies or hypothetical frequencies. According to these theories, the $\mathrm{P}(\mathrm{A})$ is the relative frequency of $\mathrm{A}$ in either an idealized actual or hypothetical long series of repetitions of an experiment. Interpreting the LLN this way requires a series of repetitions of experiments which are themselves series of repetitions, i.e., in von Mises terminology, a collective of collectives. In the case at hand this unfortunately yields an actual or hypothetical collective of Bohmian universes $\left\{\mathrm{Q}(\mathrm{t}), \mathrm{Q}^{\prime}(\mathrm{t}) ..\right\}$, each of which is a collective. DGZ are adamantly opposed to this sort of reading, repeatedly saying throughout their work that there is only one universe and that as a result they are not interpreting typicality as a kind of probability. Alternatively, let's try understanding the theory with objective propensity, 
a la Popper. Again we face extra-worldly implications. What could it mean for the universe as a whole to have a probabilistic disposition to occur? There is nothing outside the universe to trigger the disposition.

An instinctive negative reaction to assigning probabilities to initial conditions in a deterministic universe is natural and probably even healthy. DGZ are understandably reluctant to dub the universe probable, for it invites quasi-theological pictures of supernatural beings picking the universe out of a big urn. That said, in the philosophical literature there are now accounts of chance that do not invite such unhealthy speculations. To mention two broad classes, there are "Humean" accounts of chance and "theoretical term" accounts of chance. The most popular Humean theory is one due to Lewis 1994, although one might count Skyrms 1980 in this camp as well. A recent version of the theoretical term account is Sober's 2005 "No-Theory Theory" account of chance, although views like this have been proposed earlier (Levi 1990, Sklar 1979). Let me briefly describe each type of account and how they would understand the probabilities in Bohmian mechanics.

Lewis' theory proposes that the chance of an event or proposition is simply the real numerical value in the closed unit interval assigned to that event by the Best Theory. The Best Theory is the best systematization of nature. According to it, the fundamental laws of nature are simply the axioms of the theory that best systematizes the actual facts. "Best" means that the theory strikes the best balance available between simplicity and strength (or informativeness), where simplicity is measured with respect to the concision of the law framed in some language (according to which the natural properties correspond to atomic predicates of the language) and strength is measured with respect to how many actual facts the theory implies. The "actual facts" are usually understood in some very broadly empiricist fashion; so long as they do not include nomic or irreducibly probabilistic facts we need not be too fussy about them here. Clearly, the virtues of simplicity and strength will trade-off one another. A long list of all the outcomes of all coin tosses is not very simple, nor is the claim "some side or other came up" very informative.

In 1994 Lewis noted that probabilistic laws might be so simple and strong that they may warrant membership in the set of axioms of the Best Theory. He accommodated probabilistic laws by adding the concept of fit to an assessment of strength. Systems score different fit-values for different worlds; the fit is given by the chance the system gives to a world's history: the higher the value, the better the fit. If the Best Theory assigns a one-half chance of fair coins landing heads on coin flips, then the actual world has better fitness than a world wherein coins always land heads. On Lewis' view, actual frequencies are evidence of the chances (the better the match with actual frequencies, the better the strength of the probabilistic law) but not identical with the chances (simplicity will buy some breathing room between the chances and frequencies). And chances supervene upon patterns of events in the actual world: there are no irreducible dispositions, modalities, other-worldly ensembles, and so on. 
For reasons we need not go into, Lewis' theory ascribes only trivial chances ( 0 or 1$)$ in worlds with fundamentally deterministic laws. Many philosophers, however, have seen that his restriction to stochastic dynamical systems is both undesirable and inessential to the account. Hoefer 2005, Loewer 2001 and Meacham 2005 all modify Lewis' theory of chance to cover deterministic worlds. They each do so with an explicit goal of making sense of the probabilities one finds in classical statistical mechanics and Bohmian mechanics, among other places. On Loewer's theory, for instance, one adds non-trivial objective probabilities to a deterministic theory by placing a probability distribution over the microscopic initial conditions. Each microscopic world history then has a certain probability. These micro-probabilities then imply probabilities of macroscopic events. Thus the probability of some macroscopic event A happening at $t$ is given by this microscopic probability distribution and then conditionalizing over the entire "macroscopic" history of the world up until t. In the case of statistical mechanics, one would use the microcanonical probability distribution over the initial conditions of Newtonian mechanics and conditionalize over the thermodynamic state of the world. Applied to Bohmian mechanics, the probability distribution would be over initial configurations of Bohmian particles. Instead of the microcanonical distribution the Bohmian would use a probability distribution crafted from the measure in (6). And instead of conditionalizing on the macroscopic state of the world one would conditionalize over the quantum state of the world, i.e., the effective wavefunction. The claim, then, is that the Best System of our world includes a law or axiom that places the probability distribution crafted from the measure in (6) over initial particle configurations of the universe. Conditionalizing on this distribution, one gets non-trivial chances that (as DGZ show) match up with those given by Born's rule. The reason to expect that (3) would emerge as part of the fundamental laws is that it is both simple and remarkably strong, i.e., with it we get back all the generalizations of quantum mechanics, whereas that is not the case in a theory using only (1) and (2). With a small sacrifice in simplicity, we get back all of quantum mechanics.

Sober's No-Theory theory is motivated by pointing out that science posits many objective quantities to the world based on the role they play in science. Forces and masses in classical mechanics and energy and masses in classical or quantum mechanics are examples of such quantities. Although strict empiricists like Mach were not realists about force, strict empiricism of Machian persuasion ("operationalism") is untenable. Classically, one might be a realist about forces, and quantum mechanically, one might be a realist about energy. That is, energy and forces might be real entities in the world and not merely book-keeping devices, despite the fact that we do not directly perceive them. The important point about these quantities is that it is not usually felt necessary to endow them with some nature over and above what the science requires of them. Mass just is that quantity that behaves as the relevant laws of nature say it does.

The basic idea behind this theory is that probability in some theories is akin to energy, mass and force. Probability is a central feature of many theories. The warrant for the theories is warrant for the objective existence of probability. But like the other theoretical terms, one doesn't attribute some nature to it above and beyond what the science requires of it (hence "no theory"); rather one says that it is that quantity that 
obeys Kolmogorov's axioms and plays the role it does in the science. In the case of Bohmian mechanics, when one works through the details of Sober's theory, the macrochances will be very similar to those found in Loewer's theory.

It may not escape notice how similar the two sorts of theory are. In both cases the central idea is that chance is implicitly defined by the role it plays in science. The Humean chance is tied to the Best System and a particular theory of laws, but the idea at the end is that our best science will be part of the Best System. The chances merit their inclusion in the system because they carry their weight in science (that is, they're part of the on balance simplest and strongest system). The concept's role in science is what justifies positing an objective referent for them. And that is more or less what the No-Theory account does. The No-Theory account skips the story about the origin of the laws of nature and fits the chances to the science as we find it. But still, chance is viewed as worthwhile to posit because of its value to the science. Neither theories equate the chances with the actual frequencies. And in Loewer's version of Humean chances and Sober's version of theoretical chances, chances are typically attached to macroscopic events, making each compatible with an underlying determinism.

Both sorts of theory may satisfactorily solve our problem. On Loewer's version, the DGZ result is understood as saying that the objective chance is close to one that the relative frequencies in any Bohmian history match the probabilities given by Born's rule. This chance has empirical significance because of the Humean's connection between chance and patterns of actual facts via the Best Theory. The Humean chances may depart from the frequencies, but they are guaranteed not to depart too much from themotherwise the chance theory would not be strong. Since the theory is best, it also makes sense for these chances to constrain our rational credences. We ought not bet on atypical behavior occurring and ought to bet in accordance with Born's rule. If we could do better with some other constraints on our chances, then presumably our system is not the Best Theory. Another consequence is that the connection between typicality and scientific explanation becomes clear. We don't need to explain further why (3) holds because (3) has a high chance. ${ }^{14}$ The picture here also has obvious advantages over some of the interpretations mentioned earlier. Unlike a relative frequency interpretation, this interpretation does not require that one tie the LLN result to a larger collective of which the actual universe is a part; and unlike a propensity interpretation, this interpretation does not commit us to a trigger outside the system. ${ }^{15}$

${ }^{14}$ Incidentally, DGZ claim that a tremendously problematic by-product of their analysis for thermodynamics is that the low entropy initial condition is very atypical, or rather, the microstates compatible with it are. However, it is natural to take the low entropy initial macrostate as lawlike. If so, then one assigns chances only to microstates compatible with this nomic constraint; hence they are not unlikely after one renormalizes over only the physically possible classical initial conditions.

${ }^{15}$ If the propensity view doesn't imply the existence of a trigger, then arguably it is not much different than the No-Theory Theory described below — see Frigg and Hoefer. 
On Sober's theory, we do not have any logical tie that I can see between the frequencies and theoretical chances. Science generally employs chances that have some connection to the actual frequencies, but there is nothing in the account, as in the Humean account, building this in. As a result, it is not immediately clear how the No-Theory theorist will give the DGZ result empirical significance. The theoretical chances could vary wildly from the frequencies in principle. And if that is so, then as Frigg and Hoefer stress, the No-Theory faces a problem Lewis poses for propensity theorists. If the chance of an event is simply an unadorned number, then why should such numbers constrain rational credence? The Humean can give a kind of consequentialist argument to the effect that we can't go too far wrong setting our credences equal to the chances. The No-Theory theorist can't do this in any obvious way. Perhaps Sober could use the LLN result to bridge the gap between theoretical chances and frequencies, much as Skyrms thinks the ergodic theorem bridges the gap between chances and frequencies. Exploring that option or others is a discussion for another paper.

\section{Conclusion.}

After examining various rationales for Born's rule in a Bohmian world, we settled on DGZ's explanation as especially promising. They show that, in a certain precise sense of "most", most Bohmian universes have particle configurations such that, in measurement contexts, they are randomly distributed according to $|\psi|^{2}$. While this result seems like it should be satisfying, we saw that it lacked empirical significance so long as the probability mentioned in the LLN goes uninterpreted. Further, when supplemented with some of the most established interpretations of probability, the LLN result becomes unpalatable. Theories of chance such as Humean theories and No-Theory theories then came to the rescue (and possibly, the LLN result comes to the rescue of one of them in return). These theories posit objective chances that can be non-trivial in deterministic contexts, are arguably tied to the frequencies of the actual world, and do not require positing other worlds, irreducible dispositions, or any other metaphysically controversial entity. These theories of chance may not leave everyone satisfied. Obviously, many specific parts of each theory are controversial. Even more generally, an implicit definition of chance through its role in science may not answer some questions you want answered. If you wanted some mechanism that explained why the patterns in the actual world hold, then neither theory provides anything like this. If you wanted to find some physical magnitude out there in the world that already had lots of the features you attribute to chance, then we did not find one (like an actual frequency or a propensity or a credence). We posited one. For myself, these vices, where I think they're vices, are outweighed by the virtues of actually having a theory that fits the probabilities used in science.

\section{References}

Bacciagaluppi, Guido. 1999. "Nelsonian mechanics revisited," Found. Phys. Lett. $12,1-16$. 
Barrett, J. A.: 1995, ‘The Distribution Postulate in Bohm's Theory', Topoi 14, 45-54.

Bell, J. S.: 1987, Speakable and Unspeakable in Quantum Mechanics. Cambridge University Press, New York.

Bohm, David: 1952a, 'A Suggested Interpretation of the Quantum Theory in Terms of "Hidden” Variables, Part I', Physical Review 85, 166-179.

Bohm, David: 1952b, 'A Suggested Interpretation of the Quantum Theory in Terms of "Hidden” Variables, Part II', Physical Review 85, 180-193.

Bohm, David: 1953, 'Proof that Probability Density Approaches $|\psi| 2$ in Causal Interpretation of the Quantum Theory', Physical Review 89(2), 15.

Bohm, David and Hiley, B. J.: 1993, The Undivided Universe: An Ontological Interpretation of Quantum Theory. Routledge, New York.

Bohm, David and Vigier, J. P.: 1954, 'Model of the Causal Interpretation of Quantum Theory in Terms of a Fluid with Irregular Fluctuations', Physical Review 96(1), 208-16.

Boltzmann, Ludwig: 1966, 'Further Studies on the Thermal Equilibrium of Gas Molecules', In Stephen G. Brush (ed.), Kinetic Theory, Volume 2: Irreversible Processes, Pergamon Press Ltd., Oxford, England, pp. 88-175.

Bricmont, Jean. 2001. "Bayes, Boltzmann, and Bohm" in Chance in Physics: Foundations and Perspectives, edited by Jean Bricmont, Detlef Dürr, Maria C. Galavotti, Giancarlo Ghirardi, Francesco Petruccione, and Nino Zanghi, Lecture Notes in Physics 574, (Springer-Verlag), 3ff.

Bub, Jeffrey. 1997. Interpreting the Quantum World. Cambridge University Press, London.

Callender, Craig. 2004. "Measures, Explanation and Time: Can We Explain the Boundary Conditions of the Universe?" British Journal for the Philosophy of Science, 55, 195-217.

Cushing, James T. 1994, Quantum Mechanics: Historical Contingency and the Copenhagen Hegemony, The University of Chicago Press, Chicago.

Davidson, Mark. 1979. "A generalization of the Fenyes-Nelson stochastic model of quantum mechanics," Lett. Math. Phys. 3, 271-277.

Dürr, Detlef, Goldstein, Sheldon, and Zanghi, Nino: 1992, 'Quantum Equilibrium and the Origin of Absolute Uncertainty', Journal of Statistical Physics 67(5/6), 843-907. 
Dürr, Detlef, Goldstein, Sheldon, Tumulka, Roderich, and Nino Zanghi. 2005. "BellType Quantum Field Theories", J. Phys. A: Math. Gen. 38, R1-R43.

Dürr, Detlef, Goldstein, Sheldon, Tumulka, Roderich, and Nino Zanghì, forthcoming, "Bohmian Mechanics", Encyclopedia of Philosophy, Second Edition, edited by D. M. Borchert (Macmillan Reference).

Dürr, Detlef. 2001. "Bohmian Mechanics" in Chance in Physics: Foundations and Perspectives, edited by Jean Bricmont, Detlef Dürr, Maria C. Galavotti, Giancarlo Ghirardi, Francesco Petruccione, and Nino Zanghi, Lecture Notes in Physics 574, (Springer-Verlag), 154ff.

Earman, John and Redei, Miklos. 1996. "Why Ergodic Theory Does nOt Explain the Success of Equilibrium Statistical Mechanics" British Journal for the Philosophy of Science, 47, 63-78.

Einstein, A., 1949, "Reply to Criticisms," in Schilpp, P. A., ed., Albert Einstein, Philosopher-Scientist, Evanston, IL: Library of Living Philosophers.

Frigg, R. and Hoefer, C. this volume

Geiger H., Obermair G., Helm C. 2001. "Quantum mechanics without statistical postulates" in Quantum Communication, Computing and Measurement 3, Edited by P. Tombesi and O. Hirota, Kluwer Academic/Plenum Publishers, 2001.

Hoefer, C. "The Third Way on Objective Probability: A Skeptic's Guide to Objective Chance" forthcoming.

Howson, C. 1995. "Theories of Probability" in British Journal for the Philosophy of Science 46, 1-32.

Keller, J. B., 1953. 'Bohm's interpretation of quantum theory in terms of 'hidden' variables". Physical Review, 89:1040-1041.

Levi, I. 1990. "Chance," Philosophical Topics v.18, pp.117-149.

Lewis, D. 1994. "Humean Supervenience Debugged". Mind 103, 473-490.

Loewer, B. 2001. "Chance and Determinism" Stud. Hist. Phil. Mod. Phys., 32(4), 609620.

Maudlin, T. 2005 (draft). "What Could be Objective about Probabilities?"

Meacham, C. 2005. "Three Proposals Regarding a Theory of Chance" Philosophical Perspectives 19, 281-308. 
Nelson, E. 1985. Quantum Fluctuations. Princeton University Press, Princeton.

Pauli, W. 1953. "Remarques sur le probleme des parameters caches dans la mecanique quantique et sur la theorie de l'onde pilote" in A. George (ed.) Louis de Broglie:

Physicien et Penseur (Albin Michel, Paris), 33-42.

Peruzzi, G., and Rimini, A. 2000. "Compoundation Invariance and Bohmian Mechanics", Foundations of Physics, 30, 1445.

Potel, G., Muñoz-Aleñar, M., Barranco, F. and E. Vigezzi. 2002. "Stability properties of $|\Psi|^{2}$ in Bohmian dynamics", Physics Letters A, 299, 2-3, 125-130.

Saunders, S. 2005. "What is Probability?" in Quo Vadis Quantum Mechanics, A. Elitzur, S. Dolev, and N. Kolenda, eds., Springer-Verlag, Berlin.

Shtanov, 1997. "Origin of quantum randomness in the pilot wave quantum mechanics". CERN Preprint Server, (IUCAA-38/97), 1997.

Sklar, Lawrence, 1979. "Probability as a theoretical concept", Synthese, 40(3), 409- 414.

Sklar, Lawrence, 1993. Physics and Chance: Philosophical Issues in the Foundations of Statistical Mechanics. Cambridge University Press, New York.

Skyrms, B. 1984. Pragmatics and Empiricism. New Haven. Yale University Press.

Sober, Elliot. "Evolutionary Theory and the Reality of Macro Probabilities" in E. Eells and J. Fetzer (eds.), Probability in Science, Open Court, forthcoming.

Strevens, M. 2003. Bigger Than Chaos. Harvard University Press.

Tolman, Richard C. 1938. The Principles of Statistical Mechanics. Dover Publications, Inc., New York.

Tumulka, R. 2004. American Journal of Physics 72(9), 1220-1226.

Valentini, Antony: 1991, Signal-Locality, Uncertainty, and the Subquantum H-Theorem I', Physics Letters A 156(1,2), 5-11.

Valentini, Antony and Westman, Hans. 2004. "Dynamical Origin of Quantum Probabilities" quant-ph/0403034.

Van Lith, J. 2001. "Stir in Stillness. A Study in the Foundations of Equilibrium Statistical Mechanics", PhD-thesis, Utrecht University.

Vink, Jeroen C. 1993, 'Quantum Mechanics in Terms of Discrete Beables', Physical 
Review A 48(3), 1808-1818. 